
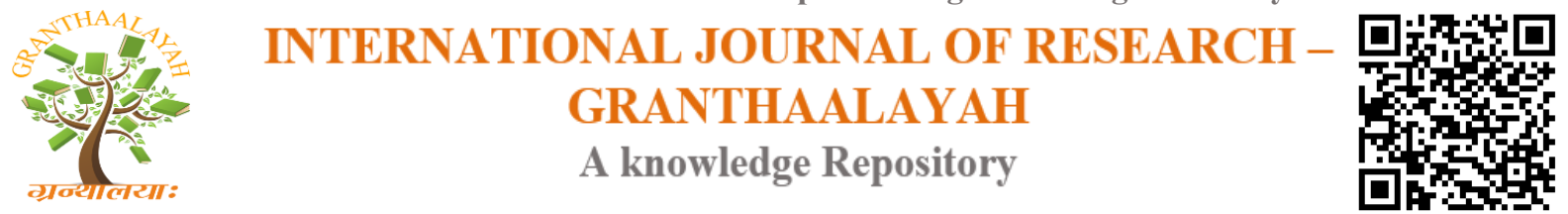

Science

\title{
AN OUTBREAK OF AVIAN PASTEURELLOSIS IN A TURKEY FARM
}

\author{
Shilpa, V.T. ${ }^{1}$, Girish, B.C. ${ }^{* 2}$, Ambujakshi T.P. ${ }^{3}$, Manjunatha, K.P. ${ }^{4}$ \\ $1,{ }^{*}, 4$ Assistant Professor, Dept. of Pathology, Veterinary College, KVAFSU, Gokula campus, \\ Vidyanagar-Post, Hassan, Karnataka, INDIA \\ ${ }^{3}$ Senior Veterinary Officer, Dept. of Pathology, Veterinary College, KVAFSU, Gokula campus, \\ Vidyanagar-Post, Hassan, Karnataka, INDIA
}

\begin{abstract}
Avian Pasteurellosis is a contagious disease of domestic and wild birds caused by Pasteurella mutocida. A turkey farm with a flock size of 500 recorded an acute death of about 102 turkeys with the history of clinical symptoms such as respiratory distress, anorexia, mucoid discharge from mouth and the nostrils, diarrhea were presented to the Department of Veterinary Pathology, Veterinary College. On thorough postmortem examination, a salient finding of petechial haemorrhages with pinpoint necrotic spots though out the liver parenchyma was observed along with pneumonic changes in lungs and vascular changes in trachea, spleen and intestines. On histopathological examination, liver revealed multifocal areas of coagulative necrosis and haemorrhages. Heart showed separation, fragmentation and degeneration of fibers with extensive inter-muscular haemorrhages. Lungs were congested with haemorrhages and pneumonic changes. Based on history, gross \& histopathological lesions and impression smear examination after staining with geimsa and methylene blue, the case was diagnosed as Avian Pasteurellosis.
\end{abstract}

Keywords:

Carrier status, liver, Pasteurella multocida, turkey.

Cite This Article: Shilpa, V.T., Girish, B.C., Ambujakshi T.P., and Manjunatha, K.P., "AN OUTBREAK OF AVIAN PASTEURELLOSIS IN A TURKEY FARM" International Journal of Research - Granthaalayah, Vol. 3, No. 11(2015): 43-46.

\section{INTRODUCTION}

Avian Pasteurellosis or fowl cholera is a contagious disease of domestic and wild birds caused by Pasteurella multocida species of bacteria. It usually occurs in septicemic form of sudden onset with high morbidity and mortality but chronic and asymptomatic infection has also been reported ((Botzler, 1991). Fowl cholera has been reported in a wide range of avian species, suggesting that all types of birds are susceptible, although host variation in susceptibility has been reported (Botzler, 1991). It has been observed that turkeys are found to be more susceptible to fowl cholera than chickens and the susceptibility to this infection increases as the age advances (Cynthia 2005). 
Chronically infected birds serve as major source of infection by disseminating the bacteria within the flock through secretions and excretions (Cynthia 2005). In the present study an outbreak of fowl cholera in turkeys has been described.

\section{MATERIALS AND METHODS}

In the present investigation, 102 dead turkeys from a flock size of 500 were presented to the Department of Veterinary Pathology, Veterinary College, Hassan with the history of clinical symptoms such as respiratory distress, anorexia, mucoid discharge from mouth and the nostrils, diarrhea and sudden death. A detailed post mortem examination of all the turkeys that were presented to the department was conducted and the gross abnormalities in various organs were noted down and recorded. Impression smears were collected from liver, pneumonic lungs and heart blood. Representative samples of organs showing gross lesions were collected in $10 \% \mathrm{NBF}$ for histopathological examination. All the samples were processed by routine paraffin embedding technique, sectioned at 4-5 $\mu$ thickness and stained with routine haematoxylin and eosin method (Luna, 1968).

\section{RESULTS AND DISCUSSION}

On thorough postmortem examination disturbances in the vascular system was evident. The observations included petechial haemorrhages with pinpoint necrotic spots throughout the parenchyma of severely congested liver. (Fig 1). Heart was congested with straw colored fluid in pericardial cavity. Few birds (5 in number) also revealed haemorrhages in the coronary blood vessels of the heart. Lungs were congested exhibiting oedema and pneumonia at various stages. Intestines showed catarrhal inflammation with congested mesentery. The mucosa of the Proventriculus showed pinpoint haemorrhages. Spleen and kidneys were congested and swollen. However, No abnormality was detected in trachea and air sacs. These observations are in line with the findings of (Cynthia 2005, Anitha Ram and Mammen J Abraham 2013).

Impression smears from liver, heart blood and pneumonic lungs revealed bipolar organism by gram's and methylene blue staining.

On histopathological examination, liver revealed multifocal areas of coagulative necrosis and haemorrhages. Heart showed separation, fragmentation and degeneration of fibers with extensive inter-muscular haemorrhages. Lungs were congested with haemorrhages and pneumonic changes. These observations are in line with the findings of (Cynthia 2005, Anitha Ram and Mammen J Abraham 2013).

Based on history, gross \& histopathological lesions and impression smear examination after staining with Geimsa and Methylene Blue, the case was diagnosed as Avian Pasteurellosis.

Owner was advised to administer the birds with sulphamethoxazole I.P. and Trimethoprim I.P. (10 and 2\% W/W, Duxprim, Neospark) @2g/L of drinking water for five consecutive days. Birds showed improvement with reduction in mortality following treatment. However, after a gap of 3 weeks mortality was observed again (up to 75 deaths) with similar disease pattern as seen previously. Although the treatment may bring the disease under control and reduce mortality, 
relapses are frequent (Cynthia 2005) once the drug is withdrawn. Reoccurrence of the disease could be due to stress related to environmental changes and carrier status prevalent in the surviving birds.

Comparative studies of flocks of chickens and turkeys either exposed or unexposed to P. multocida by Curtis \& Ollerhand (1981) and Carpenter et al. (1989) revealed that P.multocida carriers were present only in flocks that had previously experienced fowl cholera. In a longitudinal study, Pritchett et al. (1930) isolated the bacterium for up to 4 months after a fowl cholera outbreak in a chicken flock. However, it is not known if the same clone was involved because reliable methods for investigating clonality did not exist at that time. Sulong \& Maheswaran (1976) detected P. multocida by immunofluorescence in tissues of turkeys vaccinated with a virulent strain for up to 3 weeks after vaccination. These studies suggest that some birds remain carriers of P.multocida for sometime after exposure. A. P. Muhairwa (2000) reported 47\% of vaccinated broiler parents acted as carriers of P. multocida subspecies septicaon either the pharyngeal and/or cloacal mucosa. Sulong \& Maheswaran (1976) detected P. multocida by immunofluorescence in tissues of turkeys vaccinated with a virulent strain for up to 3 weeks after vaccination which suggests that some birds remain carriers of $\mathrm{P}$. multocida for some time after exposure.

\section{CONCLUSIONS AND RECOMMENDATIONS}

Avian Pasteurellosis or fowl cholera is a contagious disease of domestic and wild birds caused by Pasteurella multocida species of bacteria. In the current investigation, 102 dead turkeys from a flock size of 500 with the history of respiratory distress, anorexia, mucoid discharge, diarrhea and sudden death were presented for postmortem evaluation. Based on history, gross \& histopathological lesions and impression smear examination after staining with Geimsa and Methylene Blue, the case was diagnosed as Avian Pasteurellosis. The farmer was educated about the carrier status of the disease and was advised to cull the entire flock and start a fresh batch after employing thorough disinfection procedures. Hence, it is recommended to all poultry health professionals to adopt proper hygienic and sanitary measures to prevent Pasteurellosis as it is not economical to treat the birds during an outbreak.

\section{REFERENCES}

[1] Anitha, R. and Mammen, JA. Histopathological lesions in pasteurellosis in an emu-A case report, Indian Journal of Veterinary Pathology. 37(2), 2013, 229-230.

[2] Botzler, RG. Epizootiology of avian cholera in wild fowl, Journal of Wildlife Diseases. 27, 1991, 367-395.

[3] Carpenter, TE., Snipes, KP., Kasten, RW., Hird, DW. \& Hirsh, DC. Molecular epidemiology of Pasteurella multocida in turkeys American Journal of Veterinary Research.52, 1991, 1345-1349.

[4] Curtis, PE. and Ollerhead, GE. Investigation to determine whether healthy chickens and turkeys are oral carriers of Pasteurella multocida, Veterinary Record.108, 1981, 206- 207.

[5] Cynthia, MK. The Merck Veterinary Manual, 9th ed. Merck \& Co., New Jersey, USA; 2005, 2229.

[6] Luna, AG. Manual of Histological staining methods of the Armed Forces Institute of Pathology. 3rd ed. L.G. Megrow-Hill Book Co., New York; 1968, 32-37. 
[7] Muhairwa, A P., Christensen, JP. and Bisgaard, M. Investigations on the carrier rate of Pasteurella multocida in healthy commercial poultry flocks and flocks affected by fowl cholera, Avian Pathology. 29(2), 2000, 133-142.

[8] Pritchett, IW., Beaudette, FR. and Hughes, RW. Epidemiology of fowl cholera. Field observations of the spontaneous disease, Journal of Experimental Medicine. 52, 1930, 249-258.

[9] Sulong, AK. and Maheswaran, SK. Studies on Pasteurella multocida. Immunofluorescence detection of the organisms in spleen and lungs of turkeys vaccinated with live oral vaccines, Veterinary Microbiology. 1, 1976,3-14. 